\title{
ExpertInnen statt AktivistInnen: Der Entpolitisierungsdiskurs in der Aids-Arbeit der 1980er Jahre ${ }^{1}$
}

Peter-Paul Bänziger

\section{Einleitung}

Als Aids in den frühen I980er Jahren zum Thema wurde, gab es zunächst einflussreiche Kräfte, die auf die lange Tradition des repressiven und disziplinierenden »Kampfes gegen Seuchen « zurückgreifen wollten. Obwohl solche Vorschläge bis spät in die I98oer Jahre hinein zu vernehmen waren, wurden sie kaum umgesetzt. Um die Mitte des Jahrzehnts kam es nämlich zu einer wesentlichen Verschiebung in der vorherrschenden Wahrnehmungsweise von Aids, in deren Folge sich auch das Vorgehen der zentralen AkteurInnen grundlegend veränderte. In der Schweiz und in der Bundesrepublik Deutschland wurde diese Neuausrichtung spätestens im Frühjahr I987 für eine breite Öffentlichkeit sichtbar, als die großen bundesweiten Präventionskampagnen »STOP AIDS« beziehungsweise »Gib Aids keine Chance« gestartet wurden. Deren zentrales Merkmal bestand darin, dass nicht mehr pauschal die angeblichen »Risikogruppen« der »Homosexuellen, Fixer und Prostituierten« angesprochen, sondern die gesamte Bevölkerung für die Gefahr einer HIVÜbertragung bei konkreten »Risikopraktiken«, also bestimmten sexuellen Handlungen oder dem Tausch von Spritzen, sensibilisiert und über entsprechende Schutzmöglichkeiten informiert werden sollte. ${ }^{2}$ Für eine Zeitgeschichte des Selbst, wie wir sie mit dem vorliegenden Band anstreben, sind daran

1 | Für Anregungen und Kritik danke ich Eveline Müller, Roger Staub, Magdalena Beljan, Julia Stegmann und Marcel Streng.

2 | Vgl. Peter-Paul Bänziger, Vom Seuchen- zum Präventionskörper? Aids und Körperpolitik in der BRD und der Schweiz in den 1980er Jahren, in: Body Politics 2 (2014), Heft 3, im Erscheinen. 
zwei eng miteinander verwobene Prozesse interessant. Erstens kam es zu einer zunehmenden Verwissenschaftlichung und »Expertisierung « der AidsProblematik, die in der lautstark vorgetragenen Forderung nach einer »Entpolitisierung « des präventiven Handelns zum Ausdruck kam. Aids stellte in den Augen maßgeblicher AkteurInnen aufseiten des Staates wie auch der sogenannten Nichtregierungsorganisationen (NGOs) einen Spielball in einem als von ideologischen Interessen geprägt verstandenen politischen Raum dar. Diese »verpolitisierte« Thematik, wie es damals hieß, sollte deshalb so transformiert werden, dass eine durch »ExpertInnenwissen « gestützte, präventionsorientierte Form der Regulierung möglich wurde. Die Forderung nach einer Entpolitisierung zielte also insbesondere darauf ab, Aids nicht mehr vor dem Hintergrund spezifischer (»politischer«) Interessen zu verhandeln.

Dass Aids damit in einem anderen Sinne erst eigentlich »politisiert« wurde, scheint den ProtagonistInnen nicht aufgefallen zu sein: Am Thema Aids wurde nämlich ganz grundlegend über die Art und Weise (mit)verhandelt, wie gesellschaftliche Aushandlungsprozesse zu gestalten seien. Die Forderung nach einer Entpolitisierung von Aids darf also nicht mit dem Umstand verwechselt werden, dass die Thematik damit auf einer abstrakteren Ebene überhaupt erst zum Einsatz in einer politischen Intervention gemacht wurde. Mit anderen Worten: Dieses Beispiel zeigt deutlich, dass anhand eines Gegenstandes in der Regel auch der Raum mit konstituiert wird, in dem dieser verhandelt wird. Insbesondere wird bestimmt, wer überhaupt sprechen darf. ${ }^{3}$ Der im Zentrum der folgenden Ausführungen stehende Entpolitisierungsdiskurs ist auf dieser Ebene also, ebenso wie die davor und parallel dazu erkennbaren Politisierungsdiskurse, ${ }^{4}$ immer auch als spezifische Form der Politisierung des Gegenstandes zu betrachten.

Der zweite zentrale Prozess, der im Zusammenhang mit der Verschiebung zum Diskurs über die Risikopraktiken zu beobachten ist, bestand darin, dass die Aufforderung zu handeln nun zunehmend an die einzelnen Personen gerichtet wurde. Sie waren nun nicht mehr lediglich passive Objekte von durch staatliche oder parastaatliche Institutionen angeordneten Maßnahmen. Vielmehr wurden sie als aktive Subjekte angerufen, die sich selbst und ihr

3 | Vor diesem Hintergrund könnte man die Politisierung als spezifische Form der Thematisierung (d. i. der Umstand, dass etwas zum Thema wird, also überhaupt konstituiert wird) beschreiben, im Unterschied etwa zur »Problematisierung« als einer optimierenden, »lösungsorientierten« Form der Thematisierung, die etwa für die Wissenschaften und bestimmte therapeutische Formen kennzeichnend ist. Vgl. dazu auch Peter-Paul Bänziger/Annika Wellmann, Problemgeschichten. Zur Struktur und Genealogie massenmedialer Beratungskommunikation im späten zwanzigsten Jahrhundert, in: Psychotherapie \& Sozialwissenschaft 1 (2011), S. 99-118.

4 | Vgl. dazu etwa die Aktionen von Gruppen wie »Act Up!«. 
Umfeld vor den Risiken des eigenen Handelns schützen konnten und dies auch tun sollten. Die zuständigen Gremien und Organisationen stützten ihr Vorgehen zunehmend auf die damals in der Sozialmedizin breit diskutierten Herangehensweisen der »New Public Health«. Im Zentrum der vor diesem Hintergrund eingeleiteten Maßnahmen stand die statistische Kalkulation der epidemiologischen Risiken sowie eine Ausrichtung auf individuelle Verhaltensänderungen, die weniger aus äußerem Druck denn aus Einsicht und »Eigenverantwortung « resultieren sollten. Jede einzelne Person sollte in die Lage versetzt werden, für sich selbst entscheiden zu können, wie sie sich in bestimmten (Risiko-)Situationen zu verhalten habe. Aids lässt sich damit als eine der Thematiken beschreiben, über die eine »Gesundheitspolitik« in großem Maße umgesetzt wurde, die fundamental auf die Erziehung und Mitarbeit "präventiver Selbste ${ }^{5}$ ausgerichtet war.

Im vorliegenden Beitrag untersuche ich den Entpolitisierungsdiskurs am Beispiel der Gruppen und Organisationen, die in den I980er Jahren in der Schweiz in der Aids-Arbeit tätig waren. Ich zeige, dass die entsprechenden Argumente in den zeitgenössischen Debatten breit diskutiert und von zentralen ProtagonistInnen begrüßt und aktiv unterstützt wurden. Sie entwickelten neue Formen durch ExpertInnenwissen und -knowhow geleiteter Präventionsarbeit und förderten die »Erziehung « präventiver Selbste. Darüber hinaus wird deutlich, dass dieser Prozess nicht ohne Folgen für seine TrägerInnen selbst blieb. Im Laufe von nur wenigen Jahren veränderte sich ihr Selbstverständnis grundlegend: Nachdem sie sich ursprünglich als politische AktivistInnen beschrieben hatten, verstanden sie sich - zum Teil waren es dieselben Personen - ab Mitte der I980er Jahre überwiegend als ExpertInnen. Aus dem Handeln für die direkt Betroffenen im eigenen Umfeld wurde die durch ExpertInnenwissen gestützte (Lobby-)Arbeit professionell agierender NGOs.

Um diesen Wandel zu untersuchen, beschreibe ich im zweiten Abschnitt die Geschichte der ersten Jahre des Aids-Aktivismus in der Schweiz. Anschließend gehe ich auf die diskursiven Veränderungen ein, die sich Mitte des Jahrzehnts ereigneten, um dann im letzten Abschnitt auf die Frage zu sprechen zu kommen, wie sich diese auf die Organisationen der Aids-Arbeit und das Selbstverständnis ihrer Mitglieder ausgewirkt haben. Meine Darstellung fußt hauptsächlich auf Recherchen in den Archiven der Zürcher Aids-Hilfe, der Aids-Hilfe Schweiz und des schweizerischen Bundesamts für Gesundheit.

5 | Martin Lengwiler/Jeannette Madarász, Präventionsgeschichte als Kulturgeschichte der Gesundheitspolitik, in: dies. (Hg.), Das präventive Selbst. Eine Kulturgeschichte moderner Gesundheitspolitik, Bielefeld 2010, S. 11-28; vgl. auch Delphine Moreau, »Dispositifs de sécurité« et épidémie de sida, in: Labyrinthe 22 (2005), Heft 3, S. 101-110, hier S. 105. 


\section{Betroffenheit und sexuelle Befreiung}

Wie in anderen Regionen des deutschsprachigen Raums gab es in den I970er Jahren auch in der Schweiz eine starke Schwulenbewegung, die sich auf zahlreiche und aktive Gruppen und Organisationen stützen konnte. ${ }^{6}$ Als das Thema Aids im Verlauf des Jahres 1983 aufkam, konnte sich die Auseinandersetzung damit auf die Ressourcen einer national und international gut vernetzten und bis zu einem gewissen Grad institutionalisierten sozialen Bewegung stützen. Den seit 1972 bestehenden Homosexuellen Arbeitsgruppen Zürich (HAZ) beispielsweise, die bereits zum Zeitpunkt der Gründung auf eine lange Tradition politischer und kultureller Aktivitäten zurückblicken konnten, standen eigene Räumlichkeiten im Zentrum der Stadt zur Verfügung, die in der Aufbauphase vom Aids-Beratungstelefon und der Zürcher Aids-Hilfe (ZAH) mitbenutzt werden konnten. ${ }^{7}$ Die Aktivisten - und wenigen Aktivistinnen - aus dem Umfeld der Basisgruppen dieser Zeit konnten von den Erfahrungen vergleichbarer Organisationen und Bewegungen in den USA profitieren, da die ersten Fälle von Aids dort rund eineinhalb Jahre früher aufgetreten waren. Zudem hatte - wie auch in den Quellen immer wieder festgestellt wird - die Problematik dort innerhalb von kurzer Zeit ein sehr viel größeres Ausmaß erreicht. ${ }^{8}$

Deutlich ist in den Texten aus dem Umfeld dieser Gruppen zunächst die »politische«, auf weitreichende gesellschaftliche Veränderungen abzielende Ausrichtung der Argumentationen zu erkennen. Vor allem die Diskurse der »Sexuellen Revolution« der I96oer und I97oer Jahre stellen eine wesentliche Referenz dar. So schrieb André Ratti, bekannter TV-Moderator und Galionsfigur der frühen Aids-Kampagne in der Schweiz, dass man »leider immer noch in einer sexuell wenig aufgeklärten Gesellschaft« lebe, »das heisst die meisten Menschen betreiben zwar ausgiebig Sex, wollen aber keineswegs darüber nachdenken.« Aus diesem Grund werde Aids nicht nur von den direkt betroffenen, sondern auch von allen anderen Personen »verdrängt «. ${ }^{9}$ Das gelte, so ist in einem Thesenpapier zu einem Workshop der Aids-Hilfe Schweiz

6 | Vgl. Beat Gerber, Lila ist die Farbe des Regenbogens, Schwestern, die Farbe der Befreiung ist rot. Die Homosexuellen Arbeitsgruppen der Schweiz (HACH) von 1974-1995, Köniz 1998.

7 | Archiv der Zürcher Aids-Hilfe (ZAH), Leitbild/Strukturen, Organisationsbeschrieb, Geschichte der ZAH: Geschichte der ZAH von ihrer Gründung bis 1990 (o. J.; ca. 1992), S. 6. 8 Staatsarchiv Zürich (StAZH), WII15 2001/041.1: Studienaufenthalt in den USA und Kanada (April/Mai 1986), S. 2; StAZH, WII15 2001/041.94: integro-Gespräch über die AIDS-Problematik (16.6.1986), S. 6.

9 | StAZH, WII15 2001/041.3: Konzept für eine Strategie der Aufklärung, Information und Prävention von AIDS, sowie einiger flankierender Maßnahmen im nicht medizinischen Sektor (Sommer 1985), S. 1. 
(AHS) aus dieser Zeit zu lesen, insbesondere für anale Praktiken. Sie stellten ein »Tabu« dar, das bereits in der Kindheit im Rahmen der Hygiene-Erziehung »eingeimpft« werde - ähnlich wie die weitverbreitete Ansicht, dass die »passive « Position beim Sex zur Effeminierung der betreffenden Person führe. ${ }^{10}$ Vor diesem Hintergrund wurde verschiedentlich hervorgehoben, dass die bisher erreichten Schritte hin zu einer »freieren« Sexualität durch die AidsKampagnen auf keinen Fall gefährdet werden dürften. Unter anderem wurde beispielsweise befürchtet, dass der Ratschlag, so lange auf promiskes Sexualverhalten zu verzichten, bis verlässliches Wissen vorhanden sei, Wasser auf die Mühlen der »sexuellen Repression« leiten könnte. Damit hätten konservative Kreise die Homosexuellen nämlich »wieder genau dort, wo man uns eben haben will: Sex auf Null unterdrückt «. ${ }^{11}$ Stattdessen müsse man nun anfangen, sich von den verbreiteten und »mehr oder weniger bewussten Schuldgefühlen« zu emanzipieren; es sei höchste Zeit, endlich über Sexualität sprechen zu lernen - auch im eigenen Umfeld. Ganz im Jargon der »sexuellen Befreiung « heißt es weiter: »Wir müssen unseren Körper und unsere Sexualität bewusst leben lernen, ähnlich wie es die Frauen in ihrer Bewegung vor einigen Jahren getan haben. Wir haben ein Recht auf unseren Körper und unsere Sexualität. « ${ }^{22}$

Auch wenn es hier immer auch um den je eigenen Körper geht, enthalten solche Äußerungen zweifellos weiter reichende politische Forderungen. Zum einen bezog sich die Perspektive der AktivistInnen jener Zeit oftmals auch auf die »sexuelle Befreiung« der gesamten Gesellschaft. Zum anderen ging es ihnen um die Anerkennung ihrer sozialen Gruppen als Ganze. Die Homosexuellen seien zwar eine »tolerierte Minderheit«, heißt es beispielsweise in einem Strategiepapier der AHS vom Sommer i985, sie werde aber »von der Mehrheit der Bevölkerung keineswegs akzeptiert « ${ }^{13}$. Deutlich werden die Versuche, die Aids-Problematik mit weiteren politischen Forderungen zu verknüpfen, auch in einem Protokoll der Homosexuellen Arbeitsgruppen der Schweiz (HACH) vom März I985: »Neues Aids-Papier soll nicht nur medizinische, sondern auch politische Aspekte enthalten.« Was damit gemeint war, machen die Bemerkungen über die Planung einer Demonstration deutlich. Deren Rahmenpro-

10 | StAZH, WII15 2001/041.141: Zusammenfassung des Workshops »Sexualpraktiken« (7./8.12.1985), S. 4.

11 | Ebd., S. 2. Vgl. dazu allgemein Magdalena Beljan, Rosa Zeiten. Eine Geschichte der Subjektivierung männlicher Homosexualität in den 1970er und 1980er Jahren, Bielefeld 2014, inbesondere S. 210ff.

12 | Ebd., S. 4. Zur Frauenbewegung vgl. den Beitrag von Imke Schmincke in diesem Band.

13 | StAZH, WII15 2001/041.3: Konzept für eine Strategie der Aufklärung, Information und Prävention von AIDS, sowie einiger flankierender Maßnahmen im nicht medizinischen Sektor (Sommer 1985), S. 1. 
gramm sah zwar auch einen Diskussionsabend zum Thema Aids vor, für das Motto der gesamten Veranstaltung stand aber unter anderem der Slogan $\gg$ Es gibt einen schlimmeren Virus als Aids « zur Debatte. Vor dem Hintergrund der weiteren Vorschläge und der übrigen Punkte im Protokoll wird ersichtlich, dass es sich dabei mit großer Wahrscheinlichkeit um eine Anspielung auf die nach wie vor alltägliche Diskriminierung von Lesben und Schwulen gehandelt haben dürfte. ${ }^{14}$ Noch wurde Aids also keineswegs als von weiter reichenden politischen Anliegen unabhängige Thematik verhandelt, sondern als Teil des gemeinsamen Kampfes für die Emanzipation. Allerdings wird hier schon eine Tendenz sichtbar, vor allem »homosexuelle« Partikularinteressen zu verfolgen.

Zudem dürfte eine nicht geringe Zahl der damals Aktiven weniger aus grundsätzlichen Überzeugungen heraus gehandelt haben denn aus direkter Betroffenheit. Sie drückt sich beispielsweise darin aus, dass in der Frühzeit der Auseinandersetzung mit Aids in der Regel in der ersten Person Plural geschrieben wurde. Es ging zunächst darum, die immer zahlreicheren positiv getesteten und kranken Personen im eigenen Umfeld, also die direkt von HIV und Aids betroffenen, zu unterstützen. Wie Roger Staub, einer der wichtigsten Exponenten der Aids-Arbeit in der Schweiz im Mai 1986 betonte, herrschten zwar keineswegs vergleichbare Zustände wie etwa in San Francisco, das er auf einer Studienreise besucht hatte: »Ich hatte streckenweise den Eindruck, dass alle psychischen und menschlichen Ressourcen in der Stadt gebündelt worden sind, vereint in dem Bemühen, die tragischen Folgen dieser Krankheit, den immensen menschlichen Schmerz und die Verzweiflung aufzufangen. $\ll^{15}$ Trotzdem war auch in der Schweiz die Situation teilweise dramatisch und über den dringlichen Handlungsbedarf gab es keinen Zweifel, wie eine Gruppe von Mitgliedern der »Lederszene« aus Lausanne schrieb: »Auch in Sachen Aids sind wir durch einen Fall in unserem Kreise mit diesem Problem konfrontiert worden und sind uns daher bewusst, dass etwas getan werden muss und kann, speziell innerhalb unserer Szene. $\aleph^{16} \mathrm{Zu}$ den konkreten Forderungen, für die man sich in diesem Zeitraum einsetzte, gehörte nicht zuletzt jene nach dem Zugang zu anonymen HIV-Tests. ${ }^{17}$ Des Weiteren sollten Selbsthilfegruppen

14 | StAZH, WII15 2001/041.3: Protokoll der HACH vom 9.3.1985, S. 2.

15 | StAZH, WII15 2001/041.1: Studienaufenthalt in USA und Kanada (April/Mai 1986), S. 2; StAZH, WII15 2001/041.94: integro-Gespräch über die AIDS-Problematik (16.6.1986), S. 6.

16 | StAZH, WII15 2001/041.140: MSC Suisse Romande, Brief an den Gründerverein der AHS (11.5.1985).

17 | StAZH, WII15 2001/041.3: Vorschlag des Gründervereins an die Gründungsversammlung (Frühjahr 1985), S. 1; StAZH, WII15 2001/041.3: Brief vom 7.6.1985. 
für direkt Betroffene und ihre Angehörigen ins Leben gerufen werden, wie es an einer zentralen Stelle im bereits erwähnten Strategiepapier Rattis heißt. ${ }^{18}$

Schon früh wurden jedoch auch die Nachteile der Ausrichtung der AidsArbeit in dieser Phase diskutiert. Man wandte sich zwar nicht - oder zumindest nicht explizit - gegen die eigene Betroffenheit als Ausgangspunkt und Faktor der Mobilisierung. Hingegen verschob sich nun zusehends die Argumentationsweise: Gerade weil man so viel Leiden gesehen und betreut, so große Ängste habe, sei man zur Einsicht gelangt, dass eine grundlegend neue Strategie notwendig sei. ${ }^{19}$ Die Kritik an einer sich lediglich auf die direkt Betroffenen ausrichtenden Vorgehensweise kam aus zwei Richtungen. Erstens wurde zunehmend deutlich, dass die Beschränkung auf das »Wir« der homosexuellen/schwulen Community auf die Dauer nicht haltbar war. Tatsächlich waren in dieser Periode fast nur Mitglieder von Homosexuellen-Organisationen an der offiziellen Aids-Arbeit in der Schweiz beteiligt. ${ }^{20}$ Es ist deshalb nicht erstaunlich, dass die »Öffentlichkeitsarbeit« in Rattis Papier erst an vorletzter Stelle - nach verschiedenen Vorschlägen zur Information der eigenen sozialen Gruppe - genannt wird. ${ }^{21}$ Aus weiteren Schreiben vom Frühjahr I985 lässt sich darüber hinaus ersehen, dass mit der Öffentlichkeitsarbeit wohl in erster Linie Kampagnen gegen die Stigmatisierung und Diskriminierung der Homosexuellen gemeint waren. Ganz im Sinne des Diskurses über die Risikogruppen sollten also in erster Linie Personen informiert werden, die bereits positiv auf HIV getestet worden waren oder Aids hatten, sowie »die Schwulen«; die Informationen für »Nichtschwule « sollten vornehmlich an das medizinische Personal gerichtet werden..$^{22}$ Entsprechend betrachtete und förderte auch das Bundesamt für Gesundheitswesen (BAG) die AHS anfangs als einen »Verein, welcher sich der AIDS-Problematik bei den Homosexuellen annimmt «. ${ }^{23}$

18 | StAZH, WII15 2001/041.3: Konzept für eine Strategie der Aufklärung, Information und Prävention von AIDS, sowie einiger flankierender Maßnahmen im nicht medizinischen Sektor (Sommer 1985), S. 3.

19 | StAZH, WII15 2001/041.1: Bericht der »ad hoc Kommission PRÄVENTION« zu Handen der Mitgliederversammlung AHS vom 27./28. September 1986 in Gwatt, S. 26.

20 | So waren bei der Gründung der AHS lediglich »alle Schwulengruppen der Schweiz« eingeladen worden (StAZH, WII15 2001/041.2: Jahresbericht 1985 des Vorstands der AHS, S. 1).

21 | StAZH, WII15 2001/041.3: Konzept für eine Strategie der Aufklärung, Information und Prävention von AIDS, sowie einiger flankierender Maßnahmen im nicht medizinischen Sektor (Sommer 1985), S. 3.

22 | StAZH, WII15 2001/041.3: Vorschlag des Gründervereins an die Gründungsversammlung (Frühjahr 1985), S. 2; StAZH, WII15 2001/041.3: Brief der HAZ (12.3.1985).

23 | StAZH, WII15 2001/041.3: Brief an die AHS (30.5.1985). 
Als Aids vermehrt auch im Zusammenhang mit intravenösem Drogenkonsum zum Thema wurde, geriet diese Ausrichtung auch innerhalb der AidsHilfen unter Beschuss. ${ }^{24}$ Exakt im selben Zeitraum stellte die Ausweitung des Katalogs der Risikogruppen auf weitere Personenkreise wie Sexarbeiterinnen und (heterosexuelle) Freier auch das wichtigste Thema in der medialen Auseinandersetzung mit Aids dar. ${ }^{25}$ Nicht zuletzt auch vor diesem Hintergrund dürfte es für die AHS immer dringlicher geworden sein, ihre Basis zu verbreitern und auch die Drogen-Thematik anzugehen. Schon zwei Monate nach der Gründung fragte sich der Vorstand, ob nicht Drogenberatungsstellen mit denselben Problemen konfrontiert sein könnten. Noch im selben Jahr trat schließlich der Verein Schweizer Drogenfachleute der Organisation bei. ${ }^{26}$ Zusätzlich wurde diskutiert, ob die AHS auch Selbsthilfegruppen für »Fixer« initiieren solle. ${ }^{27}$ Dass die Problematik der einseitigen Ausrichtung der Beratungstätigkeit allerdings nicht so schnell aufgelöst werden konnte, beweist ein Brief aus dem Jahr i987. Eine Frau, die sich selbst als »Gelegenheitsfixer« bezeichnete, beklagte sich: »Auf alle Fälle wusste der >Typ < bei der >Aidshilfe < nicht so recht, was er eigentlich mit uns anfangen sollte. Seine sonstige $>\mathrm{Kundschaft}<$ sei bisher nur homosexuell. $\ll^{28}$

Ein zweiter interner Kritikpunkt an der Aids-Arbeit in den frühen Jahren bestand darin, dass man sich bisher zu sehr auf die Infizierten und Kranken konzentriert habe: »Aids-Hilfe kann eben nicht nur bedeuten: Hilfe für Kranke, Betreuung von Seropositiven, Beratung von Verunsicherten. Beraten und betreut werden heute eigentlich nur jene Menschen, die zu uns kommen. ${ }^{29}$ Mit dieser Diagnose äußerte die »ad hoc Kommission PRÄVENTION« der AHS mehr oder weniger direkte Kritik an den eigentlichen Grundlagen der älteren Strategie. Im Zuge der oben beschriebenen Ausrichtung der Kampagnen auf eine Politik der sexuellen Befreiung hatte man insbesondere darauf verzichten wollen, die Betroffenen als passive Objekte »neuer Normen« und Vorschriften anzusprechen. Stattdessen sollten lediglich Informations- und Beratungsangebote zur Verfügung gestellt werden, von denen »der Einzelne Gebrauch

24 | StAZH, WII15 2001/041.141: Zusammenfassung des Workshops »Sexualpraktiken« (7./8.12.1985), S. 4.

25 | Vgl. Bänziger, Vom Seuchen- zum Präventionskörper, Abschnitt 5.

26 | StAZH, WII15 2001/041.2: Protokoll der Vorstands-Sitzung der AHS vom 17. August 1985, S. 3.

27 | Ebd., S. 1.

28 | StAZH, WII15 2001/041.79: Brief an die AHS (1.2.1987), S. 1.

29 | StAZH, WII15 2001/041.1: Bericht der »ad hoc Kommission PRÄVENTION« zu Handen der Mitgliederversammlung AHS vom 27./28. September 1986 in Gwatt, S. 1 (Hervorh. i. 0.). 
machen kann oder auch nicht. Er bleibt handelndes Subjekt. «0 Zu welchen Verschiebungen es im Bezug auf diese klassisch »liberale« Position im Umgang mit dem Körper ${ }^{31}$ in der Folgezeit kam, beschreibe ich in den folgenden Abschnitten.

\section{Prävention und Eigenverantwortung}

Dass man sich in der Aids-Arbeit ähnlich wie in der Vergangenheit im »Kampf« gegen andere sexuell übertragbare Krankheiten auf repressive Maßnahmen stützen könnte, stand Mitte der I980er Jahre kaum mehr zur Debatte. ${ }^{32}$ Erstens kannten viele Betroffene und ihre UnterstützerInnen die Folgen der oftmals mit solchen Vorgehensweisen einhergehenden Diskriminierungs- und Stigmatisierungstendenzen nur zu gut. Zweitens gab es mit der Drogenpolitik der I980er Jahre ein anschauliches Beispiel für die Effekte repressiven Vorgehens. Gerade in Zürich, wo die Fäden der schweizerischen Aids-Arbeit zunehmend zusammenliefen, waren die negativen Auswirkungen dieser Politik kaum mehr übersehbar. In den von mir untersuchten Quellentexten sind denn auch immer wieder Hinweise darauf zu finden.

Seit Mitte der I970er Jahre war die Angst um den Verfall der »bürgerlichen Ordnung « ein zentrales Moment der schweizerischen Politik. Dies zeigte sich auch in der repressiven Ausrichtung der Drogenpolitik: So war es beispielsweise verboten - und dies obwohl das Risiko einer HIV-Übertragung durch mehrfach benutzte Spritzen längst bekannt war -, saubere Spritzen abzugeben. Aufgehoben wurde das Verbot im Kanton Zürich erst I986. ${ }^{33}$ Angesichts der Dramatik der Situation in Zürich wird dies auch nicht durch den Umstand relativiert, dass die Aufhebung des Verbots im nationalen und internationalen Vergleich eher früh erfolgte. Gefordert worden war es schon lange; in der medizinischen Praxis war es auch immer wieder öffentlichkeitswirksam unterlaufen worden. Und so ist es nicht erstaunlich, dass es im erwähnten Bericht der Präventionskommission der AHS heißt: »Als schlechtes Beispiel für repressive

30 | StAZH, WII15 2001/041.141: Zusammenfassung des Workshops »Sexualpraktiken« (7./8.12.1985), S. 3 (Hervorh. i. O.).

31 | Vgl. dazu nach wie vor Philipp Sarasin, Reizbare Maschinen. Eine Geschichte des Körpers 1765-1914, Frankfurt am Main 2001.

32 | Das bedeutet nicht, dass nicht - wie beispielsweise in Bayern - nach wie vor auch andere Vorgehensweisen diskutiert wurden. Umgesetzt wurden sie allerdings kaum. Vgl. Bänziger, Vom Seuchen- zum Präventionskörper, Abschnitt 5.

33 | Gertrud Vogler/Chris Bänziger, Nur saubergekämmt sind wir frei. Drogen und Politik in Zürich, Zürich 1990, S. 85. 
Präventionsmethoden in unserer Gesellschaftspolitik möchte die Kommission auf die verkrachte Drogenpolitik hinweisen. $\ll^{34}$

Wie ich im vorangehenden Abschnitt erwähnt habe, war eine Politik des erhobenen Zeigefingers, die von klaren Normen ausging und genaue Verhaltensvorschriften aufstellte, innerhalb der Aids-Hilfen verpönt. Die als Antwort darauf formulierte »liberale« Strategie, lediglich Informations- und Beratungsangebote zur Verfügung zu stellen, konnte sich jedoch nicht durchsetzen. Im Zentrum der Überlegungen standen nun vielmehr zwei eng miteinander verknüpfte Konzepte: die »Prävention« und die »Selbst-« beziehungsweise »Eigenverantwortung«. Diese Konzepte waren nicht mit »repressiven« Strategien vereinbar, wie Staub im Frühsommer I986 in einem Interview mit der Gewerkschaftszeitschrift integro unmissverständlich festhielt: »AIDS ist kein ordnungspolitisches Problem [...]. Wenn es repressiv wird, kann nicht mehr an die Eigenverantwortung appelliert werden.« ${ }^{35}$

Prävention und Eigenverantwortung waren keineswegs neu in der Debatte über Aids. Sie erfuhren jedoch Mitte der I98oer Jahre einen Bedeutungswandel. Noch im Jahresbericht I985 der AHS wurde Prävention schlicht mit der Verbreitung von Wissen über »Safer Sex« und den Gebrauch von Kondomen innerhalb der Risikogruppen gleichgesetzt. Prävention bedeutete hier Information. Zur gleichen Zeit kam es jedoch zu einer deutlichen Ausweitung der Bedeutung; aus einem nicht genauer spezifizierten Begriff wurde eine weitreichende Strategie. Spätestens ab Sommer I986 stand Prävention für einen umfassenden Ansatz der »aktiven « und »offensiven« Vermittlung von Wissen über Risiken und Verhaltensmöglichkeiten in der gesamten Bevölkerung. ${ }^{36}$ In diesem Zusammenhang wurde auch ein drittes Argument gegen repressive Vorgehensweisen vorgebracht: Sie verböten sich schon allein deshalb, weil damit »die ganze Geschichte in den Untergrund abtaucht. Wir verlören jede Übersicht über die Ausbreitung der Infektion. «7 $^{37}$

Das Präventionsdenken war also mit einer Verwissenschaftlichung des Blicks auf die Objekte der Prävention verbunden. Um in diesem Paradigma handeln zu können, musste nicht nur verlässliches und popularisierbares Wissen über die Krankheit und die Übertragungswege beziehungsweise -risiken vorhanden sein; man benötigte darüber hinaus Informationen über jene, die

34 | StAZH, WII15 2001/041.141: Zusammenfassung des Workshops »Sexualpraktiken« (7./8.12.1985), S. 8.

35 | StAZH, WII15 2001/041.94: integro-Gespräch über die AIDS-Problematik (16.6.1986), S. 10.

36 | StAZH, WII15 2001/041.1: Bericht der »ad hoc Kommission PRÄVENTION « zu Handen der Mitgliederversammlung AHS vom 27./28. September 1986 in Gwatt, S. 2, 4f. und 26.

37 | Ebd., S. 8. 
mit diesem Wissen angesprochen werden sollten. Im Vergleich zur Zeit der direkten Betroffenheit erweiterte sich damit der Fokus. Gegenstand der Überlegungen und Risikokalkulationen war nun die gesamte Bevölkerung: »Es ist nämlich so: ab einer gewissen Durchseuchungsrate ist es sinnlos, noch Prävention zu betreiben. Der Aufwand steht dann in keinem Verhältnis mehr zum Erfolg ${ }^{38}$, heißt es im bereits mehrfach zitierten Bericht. Bezeichnend für diese Konzeption ist ferner der Umstand, dass der Diskurs über die Risikogruppen nicht ganz verschwand, sondern durch denjenigen über die »Zielgruppen « ${ }^{39}$ ersetzt wurde: Es war nun nicht mehr pauschal von »den Homosexuellen« oder »den Fixern« die Rede. Stattdessen wurden innerhalb des allgemeinen Rahmens der Präventionskampagnen spezifische Module für einzelne Gruppen erarbeitet. ${ }^{40}$

Auch der zweite zentrale Begriff des Präventionsdiskurses, die »Eigenverantwortung «, war schon früher verwendet worden. Damals hatte er allerdings noch nicht die eindeutig positive Bedeutung, die er später bekommen sollte. So wird in einem Thesenpapier aus dem Jahr 1985 klargestellt, »dass Selbstverantwortung an sich kein praktikabler Begriff ist. Selbstverantwortung ist ein Begriff aus dem liberalen Gedankengut, der davon ausgeht, dass alle Personen einer Gesellschaft die gleichen Startbedingungen für das Leben haben. Was wir ja nicht im Ernst glauben können.«Bereits hier lässt sich aber die Stoßrichtung der Debatte um die Eigenverantwortung absehen. Gleich im Anschluss an die zitierte Textstelle heißt es nämlich: »Deshalb ist >Selbstverantwortung< auch als Ziel von Aktionen anzusehen. $\aleph^{41}$ Der liberale Diskurs, der auch die Rede vom »Subjekt« prägte, konnte sich also schon damals nicht klar durchsetzen. Er wurde mit sozialpädagogischen Elementen vermischt, deren Einfluss in der Folge zunahm. In gewisser Hinsicht konnte dabei auch an den zweiten Strang der älteren Debatte, die Ausrichtung auf die »sexuelle Befreiung «, angeknüpft werden; auch darin waren pädagogisch-therapeutische Konzepte zentral gewesen. ${ }^{42}$ Die Subjekte des freien (sexuellen) Handelns mussten erst geschaffen

38 | Ebd., S. 26.

39 | StAZH, WII15 2001/041.5: Leitbild '87, S. 4.

40 | StAZH, WII15 2001/041.1: Bericht der »ad hoc Kommission PRÄVENTION« zu Handen der Mitgliederversammlung AHS vom 27./28. September 1986 in Gwatt, S. 13 und 22.

41 | StAZH, WII15 2001/041.141: Zusammenfassung des Workshops »Sexualpraktiken« (7./8.12.1985), S. 2 (Hervorh. d. Verf.).

42 | Vgl. Beljan, Rosa Zeiten, S. 83ff; Benno Gammerl, Ist frei sein normal? Männliche Homosexualitäten seit den 1960er Jahren zwischen Emanzipation und Normalisierung, in: Peter-Paul Bänziger/Magdalena Beljan/Franz X. Eder/Pascal Eitler (Hg.), Sexuelle Revolution? Zur Geschichte der Sexualität im deutschsprachigen Raum seit den 1960er Jahren, Bielefeld 2015, S. 223-243. 
werden: »Was hier gefragt ist, geht in Richtung sverhaltens-konditionierene [sic!] Erziehung $\ltimes^{4{ }^{43}}$ In Bezug auf die ursprüngliche Absicht, die zu informierenden und beratenden Personen nicht als »Objekte« zu behandeln, stellt dies in der Tat eine nicht unbedeutende Veränderung dar.

Bei der Umsetzung dieses Erziehungsprogramms sollte es nicht darum gehen, die Zielgruppen zu bestimmten Verhaltensweisen zu zwingen oder ihr Verhalten durch »Sexpolizisten« überwachen zu lassen, wie es laut der erwähnten Präventionskommission in Holland und den USA gemacht wurde. ${ }^{44}$ $»$ Mit dem Drohfinger AIDS erreichen wir niemanden ${ }^{45}$, heißt es stattdessen. »Um eine dauerhafte Verhaltensänderung herbeizuführen«, müsse »der Einzelne lernen, seine eigene Sexualität, sein eigenes Verhalten zu reflektieren. $\aleph^{46}$ Die Ausrichtung auf die einzelnen Personen blieb also erhalten, allerdings unter neuen Vorzeichen. Es ging weder um die konkret Betroffenen im eigenen Umfeld noch um die frei entscheidenden Subjekte des liberalen Diskurses. Vielmehr sollte jede einzelne Person in der Bevölkerung zu einem präventiven Selbst erzogen werden. Um dieses Ziel zu erreichen, so wurde immer wieder betont, dürfe man nicht mit der Angst vor Aids und HIV operieren. Wichtig sei hingegen, »Vertrauen « zu schaffen. ${ }^{47}$ Einer der zentralen Aspekte einer solchen Strategie müsse zudem darin bestehen, Sexualität positiv darzustellen: »Wir müssen das umdrehen, die Botschaft positiv, witzig, lustbetont verpacken. Nur auf diese Art können wir in der Szene ankommen. « $^{48}$ Eine der ersten Kampagnen, die sich auf dieses Programm stützte, war die Einführung und Vermarktung des als besonders sicher geltenden »Hot Rubber«-Kondoms, die bereits im Sommer I9 85 auf breiter Basis erfolgt war. Ab I9 86 wurde sie in vielen europäischen Ländern übernommen, beispielsweise von der Deutschen Aids-Hilfe. ${ }^{49}$

\section{Von Aktivistinnen zu Expertinnen}

Wie ich bereits gezeigt habe, wurde immer wieder auf die Drogenpolitik verwiesen, wenn ein Beispiel für die negativen Auswirkungen repressiven Vor-

43 | StAZH, WII15 2001/041.1: Bericht der »ad hoc Kommission PRÄVENTION« zu Handen der Mitgliederversammlung AHS vom 27./28. September 1986 in Gwatt, S. $21 f$.

44 | Ebd., S. 23.

45 | Ebd., S. 13.

46 | Ebd., S. 21.

47 | Ebd., S. 8.

48 | Ebd., S. 13.

49 | Ebd., S. 14. Zur Geschichte von Aids in der BRD vgl. Bänziger, Vom Seuchen- zum Präventionskörper; Beljan, Rosa Zeiten, S. 173ff. 
gehens benötigt wurde, obwohl sich auch hier Mitte der I980er Jahre ein (weniger weitreichender) Paradigmenwechsel abzuzeichnen begann. ${ }^{50} \gg$ Das Drogenproblem «, so lautet die Fortsetzung der oben zitierten Textstelle über die »verkrachte « Drogenthematik, werde »mehr und mehr verwaltet. Es sind mehr Leute durch das Drogenproblem beschäftigt, als Suchtkranke vorhanden sind. $\aleph^{51}$ Vor dem Hintergrund der zeitgenössischen Diskussionen ist die Annahme plausibel, dass hier auf die bis heute andauernde Tendenz zur Medikalisierung angespielt wurde - freilich ohne zu bemerken, dass gerade die Rede von den »Suchtkranken« zentraler Bestandteil des entsprechenden Diskurses war. Wer süchtig und krank ist, entspricht in keiner Weise dem Ideal jener eigenverantwortlich handelnden, sexuell aktiven Person, die das Präventionsdenken zum Ziel hatte. Auch die Kritik an der Ausrichtung auf die intensive Betreuung der direkt Betroffenen findet sich hier wieder.

Gleichwohl hinkt der von den ExponentInnen der Aids-Arbeit aufgestellte Vergleich mit der Drogenthematik in zweierlei Hinsicht. Zum einen stellt auch die Medikalisierung eine Form der Expertisierung und Entpolitisierung dar. Der Unterschied liegt einzig in der Form, welche diese annimmt: hier die Verwaltung einzelner »Kranker« durch einen großen staatlichen (Fürsorge-) Apparat, dort die auf allgemeine Verhaltensänderungen abzielende Präventionskampagne der »schlanken« AHS, deren Vertreter - und zunehmend auch Vertreterinnen ${ }^{52}$ - die »Professionalität« und die »Effizienz«ins Zentrum ihrer Bemühungen stellten und die Arbeit immer stärker auf die Medienwirksamkeit auszurichten begannen. ${ }^{53}$ Zum anderen gab es auch in der Drogenthematik verschiedene Bestrebungen, die sich im Rahmen des Präventionsdiskurses verorten lassen, während die Betreuung der direkt Betroffenen auch im Zu-

50 | Vgl. dazu Jakob Tanner, Vom schwierigen Umgang mit Drogen in der Konsumgesellschaft. Die Rehabilitationszentren Aebi-Hus/Maison Blanche im Kontext der schweizerischen Gesellschaftsentwicklung, in: Roland Baer, Drogenhilfe zwischen Rausch und Nüchternheit. Suchttheorie, Drogenpolitik und Rehabilitationsalltag am Beispiel des Aebi-Hus/Maison Blanche 1974-1999, Bern u. a. 2000, S. 237-262, hier S. 243 und 255f.; Daniela Oertle, Räumliche Interventionen der Zürcher Stadtbehörden gegen die offene Drogenszene von 1989 bis 1995. Auflösung der Drogenszene und Überlagerung der städtischen Drogenpolitik mit der Asyldebatte, Lizenziatsarbeit der Philosophischen Fakultät der Universität Zürich 2010.

51 | StAZH, WII15 2001/041.1: Bericht der »ad hoc Kommission PRÄVENTION « zu Handen der Mitgliederversammlung AHS vom 27./28. September 1986 in Gwatt, S. 8.

52 | StAZH, WII15 2001/041.94: integro-Gespräch über die AIDS-Problematik (16.6.1986), S. 4.

53 | Vgl. Peter-Paul Bänziger, Konstellationen und Koalitionen im Sprechen über Aids in den 1980er Jahren, in Achim Landwehr (Hg.), Diskursiver Wandel, Wiesbaden 2010, S. 31-51, hier S. 43-45. 
sammenhang mit Aids nach wie vor wichtig blieb. Wenn die VertreterInnen der AHS auf die Drogenpolitik hinwiesen, verglichen sie also oftmals unterschiedliche Handlungsfelder und vergaßen zudem, dass die Betreuungsarbeit an die lokalen Tochterorganisationen, die kantonalen Aids-Hilfen wie die ZAH, delegiert worden war.

Mit der Neuausrichtung der Beschäftigung mit Aids ging denn auch eine deutliche Veränderung der Struktur der beteiligten Organisationen einher. Zu Beginn war die Aids-Arbeit in der Schweiz - ähnlich wie in den USA ${ }^{54}$ fast ausschließlich von Interessengruppen wie der Loge 70, dem »Verein für schwulen Fetisch und Leder in der Schweiz«, und weiteren Organisationen mit großer Nähe zur Basis getragen worden. ${ }^{55}$ Die oben erwähnte »Hot Rubber«Kampagne beispielsweise wurde von der »Aids-Gruppe« der HAZ konzipiert und organisiert. Man kann diese Gruppen als »lokale« oder »Basisorganisationen« beschreiben, die meistens lokal tätig sind, autonom handeln können und eine weitgehend horizontale Organisationsstruktur aufweisen. Sie setzen sich hauptsächlich aus mehr oder weniger direkt Betroffenen zusammen oder arbeiten eng mit diesen und ihrem Umfeld zusammen. ${ }^{56}$

Während der hier im Zentrum stehenden Phase zwischen ca. I985 bis I987 lässt sich beobachten, dass sich die Aids-Arbeit immer stärker zur AHS und ihren lokalen Tochterorganisationen verschob. Im Zuge dieses Prozesses kam es auch zu einer »Professionalisierung « der AHS selbst, die nun zunehmend die typische Rolle einer $\mathrm{NGO}^{57}$ übernahm. Unmissverständlich heißt es im »Leitbild '87《: »Die Aidshilfe Schweiz ist politisch neutral, überkonfessionell und in keiner weise [sic!] Sprecherin anderweitiger Minderheitsanliegen. Sie macht nur aktive Aidspolitik. ${ }^{58}$ Deutlicher könnte die Abgrenzung von der oben erwähnten Forderung, die Aids-Arbeit immer auch in den Zusammenhang weitergehender politischer Forderungen zu stellen, nicht sein.

Als Vorbilder für die neue Ausrichtung werden gleich im Anschluss an die zitierte Textstelle der WWF, Amnesty International und Greenpeace genannt sowie das BAG, das für seine »Sachbezogenheit und Aufgeschlossenheit« aus-

54 | Vgl. dazu Steven Epstein, Impure Science. AIDS, Activism, and the Politics of Knowledge, Berkeley/Los Angeles/London 1996, S. 8.

55 | Sogar bei den HACH handelte es sich noch nicht um eine hauptsächlich konkreten Projekten und der Lobbyarbeit verpflichtete NGO, vgl. Gerber, Lila ist die Farbe des Regenbogens, S. 116-122 und 128-139.

56 | Vgl. Eveline Müller, Las Mélidas. Zwischen revolutionärer Bewegung und institutionalisierter NGO. Veränderungen, Schwierigkeiten und Möglichkeiten einer lokalen feministischen Organisation in El Salvador, Lizenziatsarbeit in Ethnologie an der Philosophischen Fakultät der Universität Zürich 2007, S. 4 und 48ff.

$\mathbf{5 7}$ | Vgl. dazu ebd., S. 4 und $44 f$.

58 | StAZH, WII15 2001/041.5: Leitbild '87, S. 3. 
drücklich gelobt wird. Hatten die Neuen Sozialen Bewegungen noch die Distanz zu staatlichen Einrichtungen betont, so wurden die aus ihnen hervorgegangenen Organisationen der Aids-Arbeit nun zunehmend selbst zum Teil der Administration: Der Staat bekam Mitspracherechte, wurde zum maßgeblichen Geldgeber und »Partner«, mit dem sie eng zusammenarbeiteten. Im gleichen Zuge überließ er ihnen bestimmte Aufgaben und konnte sich so den Anschein einer »zivilgesellschaftlichen« Öffnung geben, was letztlich dazu führte, dass Teile der Aids-Arbeit gerade der politischen/öffentlichen Kontrolle entzogen wurden. Neben den institutionellen Eigendynamiken innerhalb der AHS dürfte diese Entwicklung vor allem durch die finanzielle Förderung der AHS durch das Bundesamt und die personelle Verflechtung der beteiligten Organisationen unterstützt worden sein. ${ }^{59}$ Der Begriff Nichtregierungsorganisation ist hier folglich - wie in vielen anderen Fällen auch - irreführend und verschleiert ihre gouvernementale Funktion. ${ }^{60}$

Doch neben solchen institutionen- und bewegungsgeschichtlichen Argumenten sollte auch die Rolle des Präventionsdiskurses und die damit einhergehende Veränderung des Selbstverständnisses von dessen PropagandistInnen nicht unterschätzt werden. Diese verabschiedeten sich langsam von der Basis. Wie ich oben dargestellt habe, verloren Aussagen über die Betroffenheit und die Unfassbarkeit von Aids an Bedeutung. Zunehmend ersetzten Projektorientierung sowie strategische und ökonomische Abwägungen den politischen Aktivismus; die AkteurInnen beschrieben sich nun als ExpertInnen und argumentierten folgerichtig: »Unsere Mitarbeiter werden weniger aufgrund ihres >wollens $<$ als vielmehr ihre >könnens $<$ bestimmt. $\ll^{61}$ Auch wenn Staub im zitierten integro-Interview betonte, dass die Aids-Arbeit keineswegs »verexpertisiert « ${ }^{62}$ werden solle und mit den hauptsächlich von Freiwilligen getragenen lokalen Aids-Hilfen der Basisbezug tatsächlich auch weiterhin bestand, lässt sich ohne Weiteres auf die Schweiz übertragen, was Steven Epstein über die USA schreibt: »The AIDS movement can best be compared with the relatively short list of movements that neither simply enlist experts nor attack them but, rather, undergo the process of >expertification $<. «{ }^{63}$ Zudem durchliefen auch die »basisnahen« lokalen Aids-Hilfen einen Prozess der Professiona-

59 | Vgl. auch Bänziger, Konstellationen und Koalitionen, S. 45-49.

60 | Vgl. Dirk Messner, Politik im Wandel. NGOs in der Irrelevanzfalle oder NGOisierung der (Welt-)Politik?, in: Friedrich Ebert Stiftung (Hg.), Globale Trends und internationale Zivilgesellschaft oder: Die NGOisierung der (Welt-)Politik?, Bonn 1996, S. 11-38, insbesondere S. 15-20.

61 | StAZH, WII15 2001/041.5: Leitbild '87, S. 8.

62 | StAZH, WII15 2001/041.94: integro-Gespräch über die AIDS-Problematik (16.6.1986), S. 4; vgl. auch ebd., S. 16.

63 | Epstein, Impure Science, S. 13. 
lisierung, wie die Auswertung des Archivs der ZAH zeigt. Nicht wenige von ihnen waren zudem erst auf Anregung der nationalen Dachorganisation, also »top-down«, gegründet worden. Sie waren folglich nur teilweise aus lokalen Strukturen entstanden, wenn sich auch ihre MitarbeiterInnen nach wie vor großenteils aus dem Kreis der mehr oder weniger direkt Betroffenen rekrutierten.

Die Umstrukturierung der schweizerischen Aids-Arbeit wurde nicht von allen begrüßt. Am deutlichsten wird die Kritik in einem Brief, in dem in detaillierter Weise der Austritt aus der ZAH begründet wird. Der Verfasser, ein Sozialarbeiter aus dem Bereich der Drogenarbeit, argumentierte zunächst ganz im Sinne des Präventionsdiskurses: »Ich persönlich habe kein Interesse daran, das Thema Aids als Verwaltungsgebiet zu betrachten, Aids-Patienten zu verwalten und zu bevormunden. Es ist wieder dasselbe alte Lied, alles schreit nach Kontrolle.« Stattdessen müsse es darum gehen, die gesamte Bevölkerung zu mobilisieren, während die Betreuung der Kranken ruhig den bestehenden sozialstaatlichen Einrichtungen überlassen werden könne. Im Gegensatz zur Aids-Hilfe verfügten sie über die Mittel, diese Arbeit auch tatsächlich zu leisten. Auch die Kritik an den schwerfälligen Verwaltungsapparaten teilte er: Alles müsse nun »organisiert und strukturiert sein«.

$\mathrm{Zu}$ einem gewissen Teil mag sich diese Kritik an Problemen entzündet haben, die in Zusammenhang mit dem Abgrenzungsprozess zwischen nationalen und lokalen Aids-Hilfen entstanden. Wenn der Briefschreiber aber fortfährt, dass noch die »letzte Frage [...] im Detail auf ihre Seriosität« hin geklärt werden müsse, wird ein grundsätzliches Unbehagen an den mit der Institutionalisierung verbundenen Professionalisierungs- und Expertisierungstendenzen sichtbar. ${ }^{64}$ Er zitiert unter anderem aus einem Protokoll, in dem zu lesen sei, dass der »Aufbau einer funktionsfähigen Struktur vorrang [sic!] « ${ }^{65}$ vor konkreten Aktionen habe. Man konzentriere sich auf die Mittelbeschaffung und opfere jegliche Spontanität, um dieses Ziel nicht zu gefährden. Unter anderem sei »die Spritzenverteilung [...] mit der Begründung« abgelehnt worden, »man könne es sich nicht leisten, da man keine Machtposition hat, jetzt während den Finanzverhandlungen eine solche >provokative< Aktion zu unterstützen « ${ }^{66}$ Es sei nun endlich Zeit, sich von dieser durch eine »unterwürfige Haltung « hervorgerufenen »Selbstkastrierung « zu emanzipieren, sonst riskiere man, »zur erneuten Unterdrückung von zukünftigen Randgruppen« beizutragen. ${ }^{67}$

Deutlich ist hier die Spannung zwischen seinen Erfahrungen aus der sozialen Arbeit, seiner Sympathie für die direkten und spontanen Aktionen ei-

64 | StAZH, WII15 2001/041.2: Brief an den Vorstand der ZAH (Juni 1986), S. 2.

65 | Ebd., S. 3.

66 | Ebd., S. 4.

67 | Ebd., S. 5. 
nes »Act Up!«-Aktivismus und der Arbeit einer NGO zu sehen. Es lässt sich vermuten, dass er deshalb auch in der nationalen Organisation nicht glücklich wurde, in der er sich nun engagieren wollte. Für Staub dagegen hatte die Tatsache, dass Aids seiner Ansicht nach ein »neues Denken« erforderte und die gewohnten Bahnen der politischen Aushandlung verlassen werden mussten, im positiven Sinne »etwas Faszinierendes « ${ }^{68}$. An seiner Karriere lässt sich denn auch die Professionalisierung und Expertisierung der Aids-Arbeit in geradezu idealtypischer Form nachzeichnen: Ursprünglich als Geschäftsführer für die HAZ tätig, wurde er zunächst zum Vizepräsidenten der AHS gewählt. In dieser Funktion koordinierte er insbesondere die Zusammenarbeit mit dem BAG und nahm einen Sitz in der Fachkommission für AIDS-Fragen (EKAF) sowie im sogenannten »Kreativteam STOP AIDS« ein. Letzteres diente dazu, unter Einbezug der Werbeindustrie die nationalen Kampagnen zu planen. Nach Tätigkeiten als Delegierter für Aids-Fragen des Kantons Zürich und für eine Krankenhasse ${ }^{69}$ ist Staub seit 2002 der Leiter der Sektion »Prävention und Promotion« des BAG.

68 | StAZH, WII15 2001/041.94: integro-Gespräch über die AIDS-Problematik (16.6.1986), S. 11.

69 | Vgl. Barbara Lukesch, Roger Staub, einer der Aids-Aufklärungspioniere zieht, sich endgültig aus dem Tagesgeschäft zurück, in: Tages-Anzeiger 27 (1998). 
Bereitgestellt von | Universitaetsbibliothek Basel

Angemeldet

Heruntergeladen am | 17.09.18 17:51 